\title{
$A b$ initio electronic and optical spectra of free-base porphyrins: The role of electronic correlation
}

\author{
Maurizia Palummo, ${ }^{1,2, a)}$ Conor Hogan, ${ }^{1,2,3}$ Francesco Sottile, ${ }^{1,4}$ Paolo Bagalá, ${ }^{2}$ and \\ Angel Rubio 5 \\ ${ }^{1}$ European Theoretical Spectroscopy Facility (ETSF) \\ ${ }^{2}$ Department of Physics, University of Rome "Tor Vergata", Via della Ricerca Scientifica 1, \\ 00133 Roma, Italy \\ ${ }^{3}$ CNR-INFM-SMC, University of Rome "Tor Vergata", Via della Ricerca Scientifica 1, 00133 Roma, Italy \\ ${ }^{4}$ Laboratoire des Solides Irradies, Ecole Polytechnique, CEA/DSM, CNRS, \\ 91128 Palaiseau, France \\ ${ }^{5}$ Nano-Bio Spectroscopy Group and ETSF Scientific Development Centre, Dpto. Física de Materiales, \\ Universidad del País Vasco, Centro de Física de Materiales CSIC-UPV/EHU-MPC, Spain; DIPC, \\ Av. Tolosa 72, E-20018 San Sebastián, Spain; and Fritz-Haber-Institut der Max-Planck-Gesellschaft, \\ D-14195 Berlin-Dahlem, Germany
}

(Received 10 April 2009; accepted 24 July 2009; published online 24 August 2009)

\begin{abstract}
We present a theoretical investigation of electronic and optical properties of free-base porphyrins based on density functional theory and many-body perturbation theory. The electronic levels of free-base porphine $\left(\mathrm{H}_{2} \mathrm{P}\right)$ and its phenyl derivative, free-base tetraphenylporphyrin $\left(\mathrm{H}_{2} \mathrm{TPP}\right)$ are calculated using the $a b$ initio GW approximation for the self-energy. The approach is found to yield results that compare favorably with the available photoemission spectra. The excitonic nature of the optical peaks is revealed by solving the Bethe-Salpeter equation, which provides an accurate description of the experimental absorption spectra. The lowest triplet transition energies are in good agreement with the measured values. (C) 2009 American Institute of Physics.
\end{abstract}

[DOI: $10.1063 / 1.3204938]$

\section{INTRODUCTION}

Porphyrins constitute an important class of $\pi$ conjugated organic chromophores that play a fundamental role in numerous biological and chemical processes ${ }^{1-3}$ and have recently found wide application in developing technologies. Promising memory devices have recently been demonstrated in which porphyrins were used to functionalize nanowires. ${ }^{4-6}$ Their oligomers and solid aggregates are of growing interest for optoelectronic devices, solar cells, and light-harvesting devices, as well as having applications in nonlinear optics. ${ }^{7-12}$ It is not surprising therefore that, in addition to the numerous experimental studies appearing in the literature, several semiempirical and ab initio theoretical studies, based on time-dependent density functional theory (TDDFT) and quantum chemistry techniques, have been carried out in order to characterize the fundamental electronic and optical properties of these molecules. ${ }^{13-17}$

The UV/optical spectra of all porphryins are generally quite similar, being characterized by a number of weak bands or peaks in the optical range (the $Q$ bands), and a relatively strong band in the UV region (the Soret or $B$ band). ${ }^{18}$ The simplest interpretation of porphyrin spectra is given by the Gouterman four-orbital model, a semiempirical configuration interaction scheme involving excitations from the two highest occupied molecular orbitals (HOMOs) to the two lowest unoccupied orbitals (LUMOs). ${ }^{19}$ In spite of its success, however, not all spectral features can be explained by the model.

${ }^{a)}$ Electronic mail: maurizia.palummo@roma2.infn.it.
In fact, $a b$ initio quantum-mechanical approaches are required to gain a thorough knowledge of the excited state and photophysical properties of these molecules, which, despite the numerous technological applications, are not completely understood.

Previous quantum-chemical studies of the porphyrin class of molecules illustrated the important role played by electronic correlation in describing their excited state properties. Excitation energies of free-base porphine have been reported using a variety of techniques, including multiconfigurational second-order perturbation theory (CASPT2), ${ }^{15}$ multireference second-order perturbation theory, ${ }^{20}$ symmetry adapted cluster-configuration interaction (SAC-CI) ${ }^{21-24}$ and similarity transformed equation-of-motion coupled-cluster (STEOM-CC) approaches ${ }^{25}$ generally obtaining a precision of the order of $0.1-0.3 \mathrm{eV}$.

In the present work, we use an analogous approach based on many-body perturbation theory (MBPT) (Ref. 26) [namely the so-called GW method and the Bethe-Salpeter equation (BSE)], which achieved much success over recent years within the domain of solid-state physics, frequently yielding excitation energies within $0.1-0.3 \mathrm{eV}$ of the experimental values when applied to systems ranging from bulk to zero dimensional. ${ }^{27-33}$ The GW/BSE method has not been widely applied, however, to the study of $\pi$-conjugated low dimensional and molecular systems. Work carried out in this direction ${ }^{34-39}$ furthermore illustrated that some of the usual assumptions made in the application of the method, such as the use of LDA/GGA wave functions as a starting point ${ }^{37}$ or the Tamm-Dancoff approximation, ${ }^{40}$ are not always valid for 
molecular systems. ${ }^{41}$ The applicability to the porphyrin class of molecules is therefore far from obvious and the present study aims to further knowledge in this direction.

Besides being of general academic interest, the technique should prove to be important for investigating the excitonic character in extended porphyrin oligomers or solid aggregates, for which the use of the less computationally demanding TDDFT approach has been questioned, ${ }^{42}$ but which is naturally accessible within GW/BSE. In this work we concentrate on the electronic and optical properties of two such porphryins, namely, free-base porphine $\left(\mathrm{H}_{2} \mathrm{P}\right)$, the basic building block of all porphryins, and a phenyl derivative, tetraphenylporphyrin $\left(\mathrm{H}_{2} \mathrm{TPP}\right)$. This study aims, therefore, to be a first step toward a complete $a b$ initio analysis of the exciton character in porphyrin systems and in particular for understanding how this character changes when moving from isolated porphyrins to their oligomers or solid aggregates.

\section{METHODOLOGY}

In the present MBPT scheme, the DFT Kohn-Sham (KS) eigenvalues and eigenfunctions are used as a starting point for constructing the one-particle and two-particle Green's functions including all relevant aspects of electronic interaction and correlation. The key quantity is the electron self-energy operator, which can be evaluated very accurately for many materials in the GW approximation. ${ }^{27}$ The oneparticle Green's function describes quasiparticle (QP) excitations (i.e., the individual excitation of electrons and holes) while the two-particle Green's function describes coupled electron-hole excitations and is thus required for describing the optical spectrum. ${ }^{28}$

Results presented in this work are based on the following three-stage approach. As a first step, the geometrical structures of the two isolated molecules $\left(\mathrm{H}_{2} \mathrm{P}\right.$ and $\left.\mathrm{H}_{2} \mathrm{TPP}\right)$ are relaxed using DFT (Ref. 43) within the general gradient approximation (DFT-GGA) in the Perdew-Burke-Ernzerhof PBE parametrization functional. ${ }^{44}$ We use a plane-wave approach, as implemented in the QUANTUM- ESPRESSO package, ${ }^{45}$ with norm-conserving pseudopotentials and a kinetic energy cutoff of 70 Ry. Fictitious molecule-molecule interactions occurring in the repeated cell approach are eliminated by using, after convergence tests, a vacuum thickness of more than $10 \AA$. The relaxed geometries compare well with other similar calculations found in the literature and with the experimental data. ${ }^{46,47}$ In particular, the external phenyl groups of $\mathrm{H}_{2}$ TPP cause an in-plane distortion of the porphyrin ring, without any appreciable out-of-plane distortion, in agreement with Ref. 48. We then calculate, at the optimized geometries, all the KS eigenvalues and eigenvectors up to $15 \mathrm{eV}$ above the HOMO energy using LDA, ${ }^{49}$ in order to reach a good convergence in the excited-state calculations. The GGA and LDA eigenvalues, for the same fixed geometry, were found to be very similar, within the order of $0.02-0.03 \mathrm{eV}$.

In a second step, we perform GW calculations using the YAMBO $\operatorname{code}^{50}$ in order to obtain the real QP energies $E_{i}^{\mathrm{QP}}$ as corrections to the KS eigenvalues $E_{i}^{\mathrm{KS}}$ using the following well known expression: ${ }^{27}$

$$
E_{i}^{\mathrm{QP}}=E_{i}^{\mathrm{KS}}+\frac{1}{1-\beta_{i}}\left\langle\varphi_{i}^{\mathrm{KS}}\left|\Sigma\left(E_{i}^{\mathrm{KS}}\right)-V_{x c}\right| \varphi_{i}^{\mathrm{KS}}\right\rangle,
$$

where the index $i$ runs over the occupied (holes $h$ ) and unoccupied (electrons $e$ ) states. $\left|\varphi_{i}^{\mathrm{KS}}\right\rangle$ are the KS eigenfunctions, $\beta_{i}$ is given by

$$
\beta_{i}=\left\langle\varphi_{i}^{\mathrm{KS}}|d \Sigma / d E|_{E_{i}^{\mathrm{KS}}} \mid \varphi_{i}^{\mathrm{KS}}\right\rangle,
$$

i.e., the linear coefficient in the energy expansion of the selfenergy $\Sigma$, which is itself the product of the KS Green's function $G$ times the screened Coulomb interaction $W$ obtained within the random phase approximation (RPA). ${ }^{51} V_{x c}$ is the usual DFT exchange-correlation potential. A boxlike cutoff in the long-range Coulomb potential is used at this stage in order to simulate truly isolated molecular excited states. This technique is essential ${ }^{52}$ for reaching good convergence (below $0.1 \mathrm{eV}$ ) in the self-energy calculations.

In the final step of our approach, we calculate the optical spectra including excitonic effects and self-energy corrections by means of solving the BSE. By expanding the states over the KS basis, the solution of the BSE can be mapped onto an eigenvalue problem for the excitonic Hamiltonian: ${ }^{28,53}$

$$
H_{\mathrm{exc}}=\left(\begin{array}{cc}
H^{\mathrm{res}} & H^{\mathrm{coupl}} \\
-\left(H^{\mathrm{coupl}}\right)^{*} & -\left(H^{\mathrm{res}}\right)^{*}
\end{array}\right),
$$

where the resonant part,

$$
H^{\mathrm{res}}=\left(E_{e}^{\mathrm{QP}}-E_{h}^{\mathrm{QP}}\right) \delta_{e, e^{\prime}} \delta_{h, h^{\prime}}+\left\langle e h|K| e^{\prime} h^{\prime}\right\rangle,
$$

is Hermitian. The part in the lower right is denoted antiresonant. $K=W-2 v$ is the excitonic kernel, with $W$ and $v$ being the screened and bare Coulomb interaction, where the factor 2 comes from the spin degeneracy. ${ }^{53}$ The coupling part

$$
H^{\text {coupl }}=\left\langle e h|K| \overline{e^{\prime} h^{\prime}}\right\rangle
$$

is symmetric and describes the interaction between the resonant and antiresonant parts, or in other words, between the $e-h$ pairs at positive and negative (antipairs) energies (see Ref. 41 for a more detailed description of the notation). Here electron-hole antipairs are denoted by $\overline{e^{\prime} h^{\prime}}$, while $E_{h}^{\mathrm{QP}},|h\rangle$ and $E_{e}^{\mathrm{QP}},|e\rangle$ refer to the $\mathrm{QP}$ energies and eigenstates of the occupied and unoccupied states, respectively. As is often done in this framework, we replace the QP eigenfunctions $|e\rangle,|h\rangle$ with the KS ones $\left|\varphi_{h}^{\mathrm{KS}}\right\rangle,\left|\varphi_{e}^{\mathrm{KS}}\right\rangle$. While several works have shown that this approximation may not work well in highly anisotropic systems ${ }^{37}$ or strongly correlated materials ${ }^{54}$ the results presented below demonstrate that it is reasonable for describing the low lying excitations of systems such as the studied porphyrins, as was also illustrated elsewhere for azobenzene. ${ }^{41}$

Once the eigenvectors and eigenvalues $E_{\lambda}$ of the excitonic Hamiltonian $H_{\mathrm{exc}}$ are obtained, the photoabsorption cross section is obtained from 


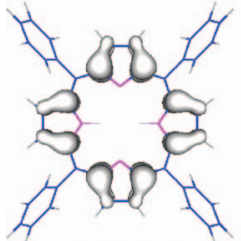

HOMO-1

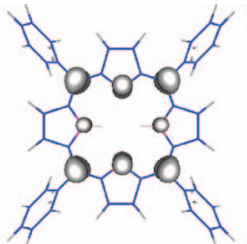

номо

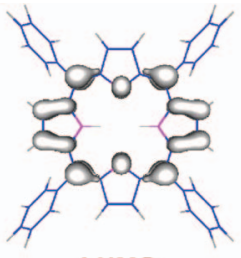

LUMO

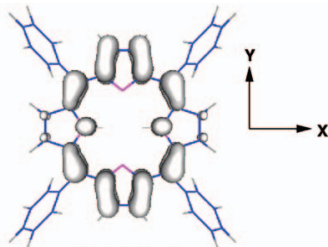

LUMO+1

$$
\sigma(\omega) \propto \sum_{\lambda}\left|D_{\lambda}\right|^{2} \delta\left(\omega-E_{\lambda}\right)
$$

where $D_{\lambda}=\langle\lambda|i \vec{\eta} \cdot \vec{r}| 0\rangle$ are the electron-hole optical strengths, $\eta$ is the light polarization direction, $|0\rangle$ is the ground state, and $|\lambda\rangle$ is the generic excited state expanded in term of electron-hole pairs and antipairs. If the electron-hole interaction is neglected, the excitations are given by vertical transitions between independent electron and hole states with $D_{\lambda}$ $=\langle h|i \vec{\eta} \cdot \vec{r}| e\rangle$, so that Eq. (6) reduces to the well known independent-particle Fermi golden rule expression.

Although the above approach is based on a local or semilocal DFT ground state calculation, it is important to realize that it does not inherit all the associated pathologies. In particular, the short-range exchange-correlation potential appearing in LDA and GGA is not present in the self-energy or, more importantly, the BSE. In these methods, the screened Coulomb interaction is used instead, yielding a correct description of the long-range $1 / r$ behavior. Thus, the BSE can in principle describe charge transfer as well as Rydberg states. ${ }^{55-57}$

\section{RESULTS}

According to the Gouterman model, the HOMO $\left(b_{1 u}\right)$, $\mathrm{HOMO}-1\left(a_{u}\right), \operatorname{LUMO}\left(b_{2 g}\right)$, and LUMO $+1\left(b_{3 g}\right)$ states are the most important ones involved in the $Q$ and $B$ optical peaks. In Fig. 1 we report probability distribution isosurfaces for each of these four states as they occur for the $\mathrm{H}_{2} \mathrm{TPP}$ molecule. The inclusion of phenyl rings does not change their character with respect to free-base porphine. We confirmed that the orbital character and ordering are consistent with other DFT-LDA calculations, ${ }^{46}$ an observation that does not change if local or semilocal exchange-correlation potentials are used. All these findings attest to the robustness of the Gouterman model in describing the order and character of these energy levels based on the symmetry of the porphyrin molecular orbitals. Nevertheless, we will show below how a proper description of the optical response and photoemission spectra requires a more sophisticated theoretical treatment beyond this simple empirical model.

In Fig. 2 we show the optical spectrum of the $\mathrm{H}_{2}$ TPP molecule obtained at the independent-particle level (or equivalently, within the RPA), where a sum over the KS transitions, according to a Fermi golden rule description, is considered. Two strong peaks are visible at 1.75 and $2.15 \mathrm{eV}$. The former peak derives from $b_{1 u} \leftrightarrow b_{2 g}$ and $b_{1 u} \leftrightarrow b_{3 g}$ transitions, while the latter derives from $a_{1 u} \leftrightarrow b_{2 g}$ and $a_{u} \leftrightarrow b_{3 g}$ transitions. The RPA optical spectrum of $\mathrm{H}_{2} \mathrm{P}$, not reported here, appears very similar with a small blueshift of the two peaks (of about $0.2 \mathrm{eV}$ ). As expected, the optical spectra obtained at this level of approximation are in complete dis- agreement with the experimental data, which feature almost forbidden $Q$ bands in the visible region and very intense $B$ bands in the near-UV region.

These results stress the need to overcome the singleparticle scheme and mix the single-particle transitions. Such mixing can be achieved by means of configuration interaction techniques, the TDDFT approach, or the present GW + BSE approach. As we will see below, the four-level mixing scheme proposed in the Gouterman model appears valid for accounting for the $Q$ bands and, to a lesser extent, the $B$ bands, in agreement with published results based on quantum-chemical or TDDFT schemes. ${ }^{16,58}$

The computed QP energies for the isolated $\mathrm{H}_{2} \mathrm{P}$ and $\mathrm{H}_{2}$ TPP molecules are compared with the KS (DFT-LDA) energies in Fig. 3. The typical linear relation that is often found between the two sets of eigenvalues in many semiconductor and insulating materials (both in bulk and in low dimensional systems) is only partially reproduced in the present molecular systems. The GW calculation opens the electronic HOMO-LUMO gap in $\mathrm{H}_{2} \mathrm{P}$ to $5 \mathrm{eV}$ (1.97 eV is the corresponding DFT-LDA gap), while a QP gap of $4.39 \mathrm{eV}$ is obtained in $\mathrm{H}_{2}$ TPP (the DFT-LDA gap being $1.75 \mathrm{eV}$ in this case). In Fig. 4 we compare the levels of the $\mathrm{H}_{2} \mathrm{P}$, obtained using the GW method, with experimental UV photoemission spectroscopy (UPS) data found in the literature. ${ }^{59}$

For comparison, we also report KS eigenvalues as obtained using a local (LDA), a semilocal (GGA), and a hybrid exchange-correlation functional [B3LYP (Ref. 60)]. As these methods do not satisfy Koopman's theorem, their eigenvalues should not be directly interpreted as electron removal/ addition energies. Nevertheless, a comparison with the GW

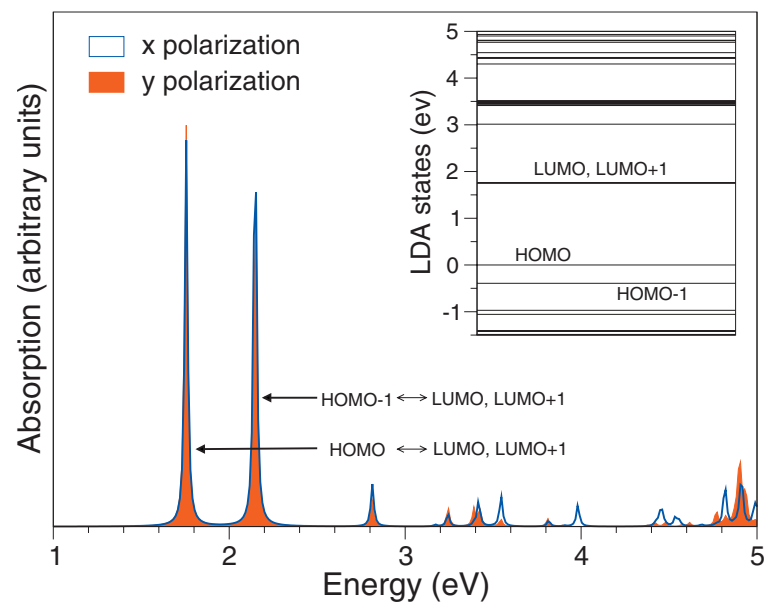

FIG. 2. Absorption spectrum of the $\mathrm{H}_{2}$ TPP molecule as obtained at the independent-particle level (RPA). Spectra according to $x$ and $y$ polarizations are almost identical. An artificial Lorenztian broadening of $10 \mathrm{meV}$ has been used. Inset: DFT-LDA energy levels. 

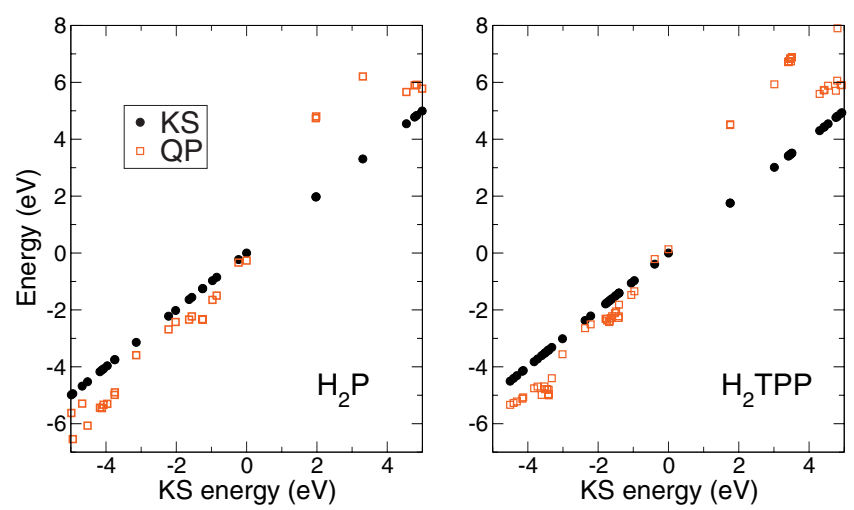

FIG. 3. Quasiparticle levels (open red squares) plotted as a function of the $\mathrm{KS}$ levels of the $\mathrm{H}_{2} \mathrm{P}$ (left panel) and the $\mathrm{H}_{2} \mathrm{TPP}$ molecules (right panel). The KS eigenvalues (filled black dots) are also reported for comparison.

eigenvalues illustrates the importance and magnitude of the self-energy corrections to the starting DFT eigenvalues (that could then be considered as an order 0 approximation to the QP energies). The theoretical description of the experimental UPS peaks is clearly much improved when QP energies are considered. It is interesting that the hybrid functional scheme also gives a very good description of the occupied states. This finding is in agreement with recent studies of porphryins $^{61}$ and of other extended carbon-based molecules. ${ }^{62,63}$

Optical spectra of the $\mathrm{H}_{2} \mathrm{P}$ and $\mathrm{H}_{2}$ TPP molecules, computed at the $\mathrm{GW}+\mathrm{BSE}$ level of approximation, are presented in the top panels of Figs. 5(a) and 5(b), respectively. The theoretical spectra are compared with the corresponding experimental data from Ref. 18 reproduced in the bottom panels. Since the computed absorption for light polarized perpendicular to the central ring is found to be negligible, we report only the in-plane $(x$ and $y)$ components. Note that vibrational coupling effects are not included in the present calculation and hence the $Q_{x}(0,1)$ and $Q_{y}(0,1)$ replicas, present in the experimental curves, are always absent in the theoretical spectra.

Inspection of Fig. 5(a) shows a reasonably good agreement between theory and experiment for the $\mathrm{H}_{2} \mathrm{P}$ molecule.

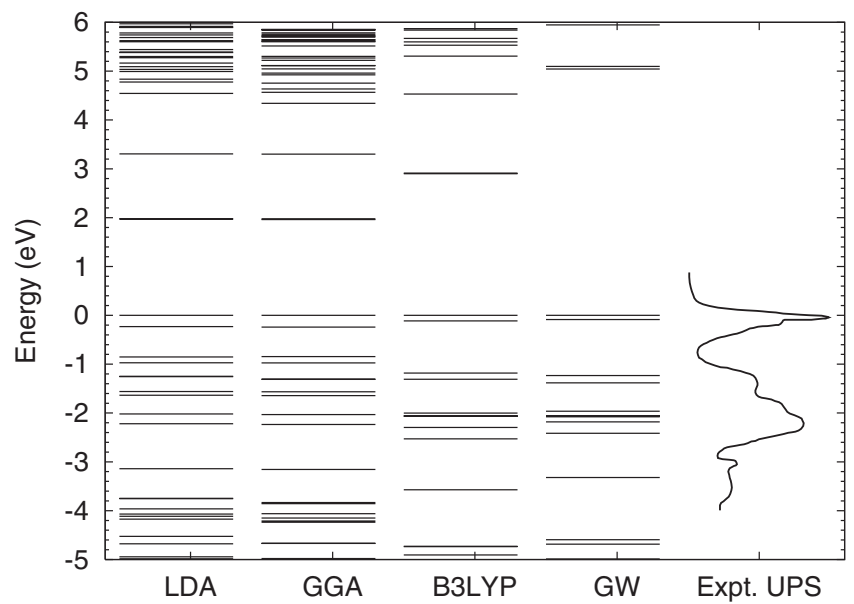

FIG. 4. Electronic states (in eV) obtained within DFT and quasiparticle schemes, compared with experimental UPS experimental data from Ref. 59. The highest occupied orbital has been aligned to zero $\mathrm{eV}$ in each case. (a) $\mathrm{H}_{2} \mathrm{P}$

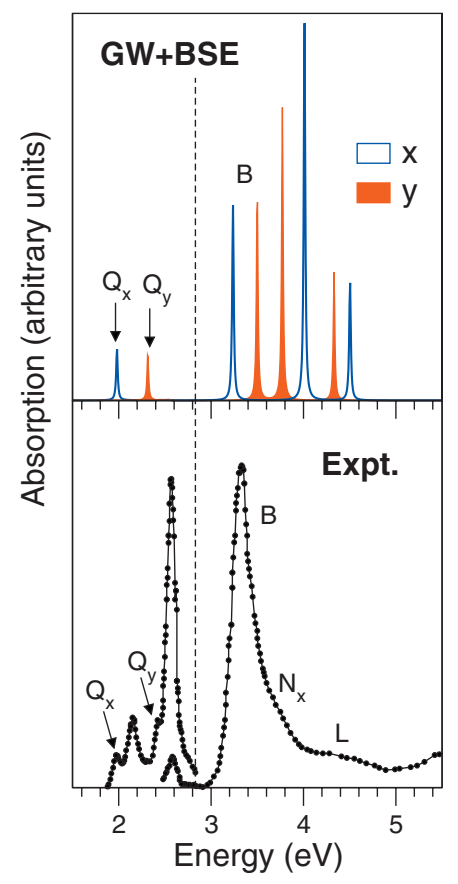

(b) $\mathrm{H}_{2} \mathrm{TPP}$

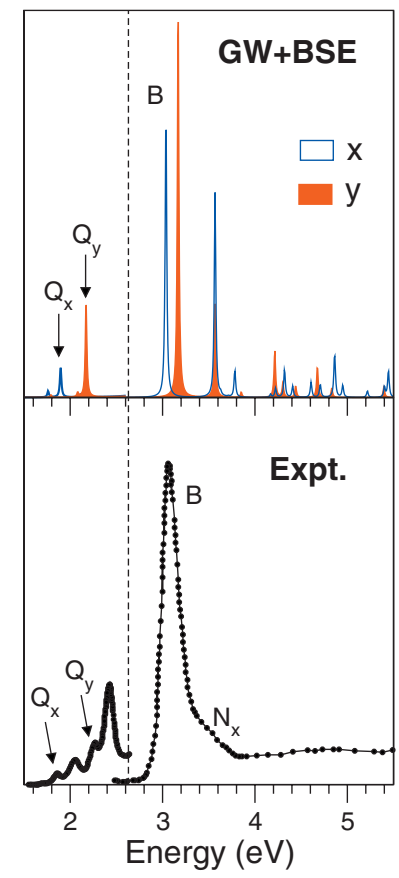

FIG. 5. Absorption spectra of the $\mathrm{H}_{2} \mathrm{P}$ (left) and $\mathrm{H}_{2} \mathrm{TPP}$ (right) molecules. The top panels show theoretical spectra obtained at the GW+BSE level of approximation for $x$ (blue) and $y$ (red, shaded) light polarizations. Experimental gas phase spectra, as reported in Ref. 18, appear in the bottom panels. Left of the vertical dashed line, the intensity of each spectrum has been multiplied by a factor of 10 for clarity. An artificial Lorenztian broadening of $10 \mathrm{meV}$ has been used.

The $Q_{x}(0,0)$ and $Q_{y}(0,0)$ peaks appear at 1.98 and $2.3 \mathrm{eV}$, respectively, in good agreement with the experimental excitation energies reported at $1.98-2.02$ and $2.33-2.42 \mathrm{eV}^{18}$ Very intense optical peaks are obtained in the UV range around $3.3 \mathrm{eV}$. Their position and shape are in reasonable agreement with the experimentally observed Soret bands at $3.13-3.33 \mathrm{eV}^{18}$ The level of accuracy reached for these low energy transitions is similar to that obtained in other ab initio approaches. Some previously computed excitation energies are reported in Table I for comparison with the GW+BSE and experimental data. Other very intense transitions are found near $4.0 \mathrm{eV}$, which may correspond to the experimentally observed $N_{x}$ and $L$ bands at 3.65 and $4.25 \mathrm{eV}$. However, the assignment is not clear in this case, as their intensities are overestimated with respect to the lower energy peaks. This may be due to a lack of convergence in the present $\mathrm{GW}$ calculations for states lying close to the continuum of molecular states: By underestimating their lifetime, the calculations yield sharper resonances that those seen experimentally. Large oscillator strengths have also been reported for the $N$ band in Ref. 21, although the accuracy of that work has been questioned. ${ }^{25}$

The comparison with experiment further improves for the $\mathrm{H}_{2}$ TPP case, where the experimental absorption spectrum of $\mathrm{H}_{2}$ TPP, shown in Fig. 5(b), is very well reproduced by the theoretical Bethe-Salpeter calculation. The $Q_{x}(0,0)$ and $Q_{y}(0,0)$ peaks appear at 1.88 and $2.15 \mathrm{eV}$, nicely reproducing the experimental transitions reported at 1.86 and 2.27 $\mathrm{eV} .{ }^{18}$ The experimentally observed Soret band is located at 
TABLE I. Excitation energies (in eV) of the $\mathrm{H}_{2} \mathrm{P}$ molecule obtained using different theoretical approaches and compared with the experimental peak positions.

\begin{tabular}{lccccc}
\hline \hline Method & Reference & $Q_{x}$ & $Q_{y}$ & $B_{x}$ & $B_{y}$ \\
\hline Expt. & 18 & $1.98-2.02$ & $2.33-2.42$ & $3.13-3.33$ & $3.13-3.33$ \\
GW+BSE & This work & 1.98 & 2.3 & 3.3 & 3.5 \\
TDDFT (LDA) & This work & 1.97 & 2.1 & 3.0 & 3.0 \\
CASPT2 & 15 & 1.63 & 2.11 & 3.08 & 3.12 \\
NEVPT2 & 24 & 2.04 & 2.51 & 3.22 & 3.30 \\
SAC-CI & 22 & 1.81 & 2.10 & 3.47 & 3.69 \\
STEOM-CC & 25 & 1.70 & 2.59 & 3.63 & 3.74 \\
TDDFT (B3LYP) & 17 & 2.27 & 2.44 & 3.33 & 3.41 \\
\hline \hline
\end{tabular}

$3.08 \mathrm{eV}$, slightly redshifted with respect to the free-base porphine, and is in very good agreement with theoretical prediction. Finally, we note that the BSE absorption curves show an optical anisotropy splitting in the $B$ band of about 0.3 and $0.15 \mathrm{eV}$ for the $\mathrm{H}_{2} \mathrm{P}$ and $\mathrm{H}_{2}$ TPP molecules, respectively.

From the calculated values of the electronic gaps $(5.0$ and $4.3 \mathrm{eV}$, for the $\mathrm{H}_{2} \mathrm{P}$ and $\mathrm{H}_{2} \mathrm{TPP}$ molecules, respectively) it is clear that the optical spectra feature strong excitonic effects, with estimated exciton binding energies of about 3.0 and $2.5 \mathrm{eV}$. This is further illustrated in Fig. 6, where we compare a GW+RPA calculation of the $\mathrm{H}_{2} \mathrm{TPP}$ absorption spectrum with the result of the full GW+BSE calculation, for the $x$-polarization. It is interesting, therefore, that the excitonic transitions associated with the $Q$ bands are found to derive from a mixing of the single particle transitions from the $\mathrm{HOMO}-1$ to $\mathrm{LUMO}+1$ and from the HOMO-1 to LUMO states, in agreement with the Gouterman model. For the $B$ and $N$ bands, however, single-particle transitions from the HOMO-2 to LUMO+1 also contribute, and hence involve states beyond the standard four involved in the Gouterman picture.

Often (and especially in extended systems) it is found that the resonant part of the excitonic Hamiltonian, $H^{\text {res }}$, is adequate for describing the optical excitations correctly. This corresponds to the so-called Tamm-Dancoff approximation ${ }^{40}$ and is equivalent to neglecting the interaction between the

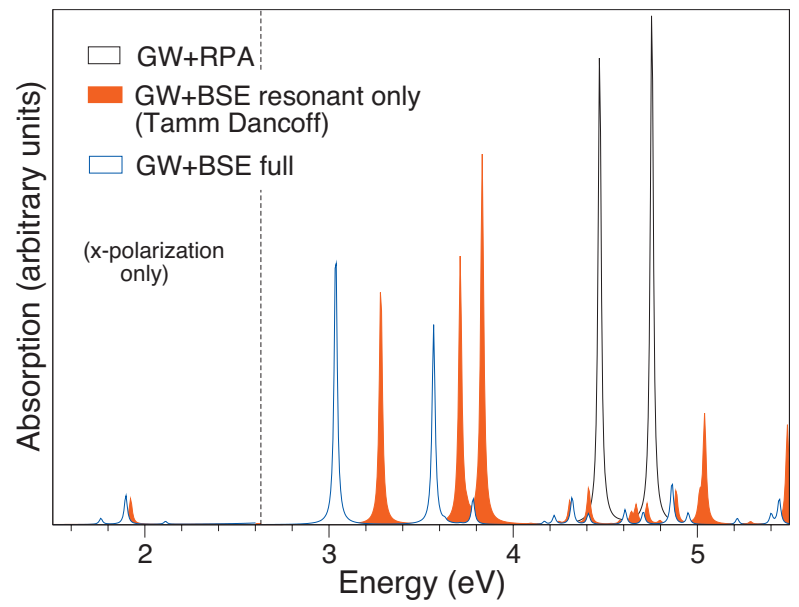

FIG. 6. Absorption spectra of the $\mathrm{H}_{2} \mathrm{TPP}$ molecule ( $x$-polarization only) computed at various levels of approximation: $\mathrm{GW}+\mathrm{RPA}$, resonant only (Tamm-Dancoff approximation), and full GW+BSE as reported previously. An artificial Lorenztian broadening of $10 \mathrm{meV}$ has been used. $e-h$ pairs and antipairs. In Fig. 6 we illustrate the influence of the coupling term $H^{\text {coupl }}$ on the final optical spectrum of $\mathrm{H}_{2}$ TPP. The effect is found to be quite large, both in the energetic peak positions and in the spectral lineshape. These findings are consistent with the conclusions of Grüning et al $^{41}$ for other carbon-based molecules and similar observations in other molecular systems. ${ }^{64}$ For this reason we have chosen to include the coupling term in all GW+BSE spectra appearing in this work.

Due to their importance in emission processes and in photobiology or medical applications such as photodynamic therapy, it is also interesting to investigate how the present approach is able to reproduce the characteristics of the triplet excitons. Within the BSE approach, triplet excitons can be calculated by simply considering $K_{e h}=W$. In Table II we compare our results for the lowest triplet excitons in both molecules with the experimental values and some of the theoretical ones as taken from the literature.

We find that the energetic position of the lowest triplet excitons appears in very good agreement with the available experimental data and with other theoretical approaches for the $\mathrm{H}_{2} \mathrm{P}$ and $\mathrm{H}_{2}$ TPP molecules. Spatial analysis of the excitonic wave function reveals that the lowest singlet and triplet excitons have different character: This is illustrated in Fig. 7 for the $\mathrm{H}_{2} \mathrm{P}$ molecule. While the lowest singlet exciton results from a mixing of the four Gouterman states (HOMO, HOMO-1, LUMO, LUMO+1), the triplet exciton is instead a pure mixing of the HOMO and LUMO states. From our previous discussion and the results shown in Tables I and II, we can conclude that the present solid-state based scheme $(\mathrm{GW}+\mathrm{BSE})$ provides singlet and triplet excitations of porphyrins to within $0.2 \mathrm{eV}$ of experiment, therefore having the very same range of accuracy as the best QC approaches discussed in the introduction.

TABLE II. Theoretical energetic positions (in eV) of the first triplet $(T)$ exciton for the $\mathrm{H}_{2} \mathrm{P}$ and $\mathrm{H}_{2} \mathrm{TPP}$ molecules. The experimental data are also reported for comparison.

\begin{tabular}{lccc}
\hline \hline Method & Reference & $\mathrm{H}_{2} \mathrm{P}$ & $\mathrm{H}_{2} \mathrm{TPP}$ \\
\hline Expt. & $65-67$ & $1.56-1.58$ & 1.45 \\
GW +BSE & This work & 1.6 & 1.5 \\
TDDFT (B3LYP) & 17 & 1.46 & \\
CASPT2 & 15 & 1.52 & \\
STEOM-CC & 25 & 1.19 & \\
QMC & 68 & 1.6 & \\
\hline
\end{tabular}



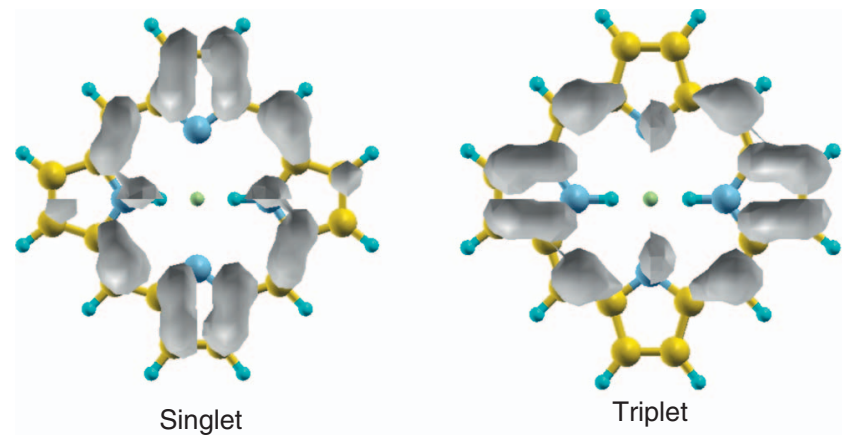

FIG. 7. Excitonic wave function of the lowest energy singlet and triplet states in the isolated $\mathrm{H}_{2} \mathrm{P}$ molecule. The green dot in the center of the molecule indicates the position of the hole.

It is useful to compare the GW+BSE results with a complementary approach. We performed a TDDFT calculation ${ }^{69}$ for the $\mathrm{H}_{2}$ TPP molecule, within the adiabatic local density approximation. The results, shown in Fig. 8 (see also Tables I and II), illustrate a reasonable agreement between the two methods for both $Q$ and Soret bands, in particular with regard to the peak positions. The relative intensities of the $x$ and $y$ polarizations are also fairly consistent between the two approaches, although we note that the TDDFT calculations appear to overestimate the relative intensity of the $Q$ and Soret bands in comparison with the experimental data.

\section{CONCLUDING REMARKS}

In conclusion, we calculated by means of first-principles MBPT the charged and neutral electronic excitations of the isolated $\mathrm{H}_{2} \mathrm{P}$ and $\mathrm{H}_{2}$ TPP molecules. The available photoemission and optical absorption measurements are well described by this approach, which also naturally provides a complete picture of the $e$ - $h$ coupling, the singlet and triplet excitations and the real space extension of the excitonic wave functions. The character and energy of the singlet and triplet lowest energy excitons turn out to be in good agreement with experiment and other $a b$ initio calculations, with accuracy comparable to quantum chemical methods. As a byproduct, we performed TDDFT calculations. The results show that a simple approximation such as ALDA can already give important insights about the optical spectrum of such
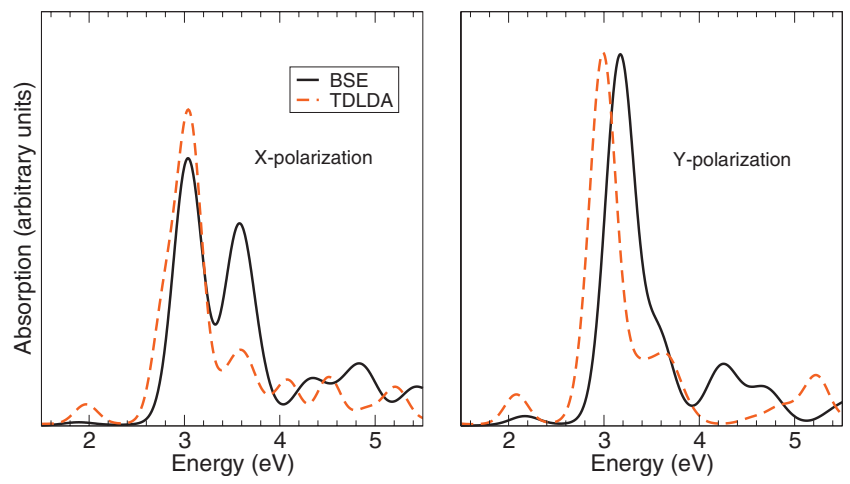

FIG. 8. Comparison of absorption spectra computed within TDDFT and BSE for the $\mathrm{H}_{2}$ TPP molecule. An artificial Lorenztian broadening of 200 meV has been used. molecules. The present study should represent the first step toward a complete ab initio analysis of the change in exciton character when moving from isolated porphyrins to their oligomers or to solid aggregates.

Note added in proof. In a recently published work, ${ }^{71}$ the GW method was also used to calculate the ionization potential of the $\mathrm{H}_{2}$ TPP molecule. The work provides further evidence that the MBPT scheme can be applied with success to the study of these molecular systems.

\section{ACKNOWLEDGMENTS}

The authors are grateful to Dr. Simona Silaghi and Dr. Norbert Esser for useful discussions, as part of the ETSF pilot user project $n$. 12. We acknowledge funding by the European Community through e-I3 ETSF project (INFRA2007-1.2.2: Grant Agreement No. 211956) and by the Spanish MEC (Grant No. FIS2007-65702-C02-01), "Grupos Consolidados UPV/EHU del Gobierno Vasco" (Grant No. IT-319-07), CSIC and by MIUR-PRIN2007. Supercomputing support is acknowledged from The Barcelona Supercomputing Center, "Red Espanola de Supercomputacion" and SGIker ARINA (UPV/EHU), and the CINECA Supercomputing Center through CNR-INFM projects (accounts cne $0 \mathrm{fm} 2 \mathrm{~h}$ and cne $0 \mathrm{fm} 2 \mathrm{v}$ ).

${ }^{1}$ M. Gouterman, in The Porphyrins, edited by D. Dolphin (Academic, New York, 1977).

${ }^{2}$ L. K. Hanson, Photochem. Photobiol. 47, 903 (1988).

${ }^{3}$ L. K. Hanson, in Chlorophylls, edited by H. Scheer (CRC, Boca Raton, 1991).

${ }^{4}$ C. Li, J. Ly, B. Lei, W. Fan, D. Zhang, J. Han, M. Mayyappan, C. Thompson, and M. Zhou, J. Phys. Chem. B 108, 9646 (2004).

${ }^{5}$ K. S. Kwok, Mater. Today 6, 20 (2003).

${ }^{6}$ X. Duan, Y. Huang, and C. M. Lieber, Nano Lett. 2, 487 (2002).

${ }^{7}$ F. J. Kampas, K. Yamashita, and J. Fajer, Nature (London) 40, 284 (1980).

${ }^{8}$ J. M. Tour, Chem. Rev. (Washington, D.C.) 96, 537 (1996).

${ }^{9}$ L. Jones II, J. S. Schumm, and J. M. Tour, J. Org. Chem. 62, 1388 (1997).

${ }^{10}$ A. Tsuda and A. Osuka, Science 293, 79 (2001).

${ }^{11}$ T. Hasobe, H. Imahori, H. Yamada, T. Sato, K. Ohkubo, and S. Fukuzumi, Nano Lett. 3, 409 (2003).

${ }^{12}$ C. B. Winkelmann, I. Ionica, X. Chevalier, G. Royal, C. Bucher, and V. Bouchiat, Nano Lett. 7, 1454 (2007).

${ }^{13}$ J. D. Baker and M. C. Zerner, Chem. Phys. Lett. 175, 192 (1990).

${ }^{14}$ I. Baraldi, A. Carnevali, G. Ponterini, and D. Vanossi, J. Mol. Struct.: THEOCHEM 333, 121 (1995).

${ }^{15}$ L. Serrano-Andrés, M. Merchán, M. Rubio, and B. O. Roos, Chem. Phys. Lett. 295, 195 (1998).

${ }^{16}$ D. Sundholm, Phys. Chem. Chem. Phys. 2, 2275 (2000).

${ }^{17}$ B. Minaev and H. Ågren, Chem. Phys. 315, 215 (2005).

${ }^{18}$ L. Edwards, D. H. Dolphin, M. Gouterman, and A. D. Adler, J. Mol. Spectrosc. 38, 16 (1971).

${ }^{19}$ M. Gouterman, J. Chem. Phys. 30, 1139 (1959).

${ }^{20}$ P. Celani and H.-J. Werner, J. Chem. Phys. 112, 5546 (2000).

${ }^{21}$ H. Nakatsuji, J. Hasegawa, and M. Hada, J. Chem. Phys. 104, 2321 (1996).

${ }^{22}$ O. Kitao, H. Ushiyama, and N. Miura, J. Chem. Phys. 110, 2936 (1999).

${ }^{23}$ J. Hasegawa, K. Takata, T. Miyahara, S. Neya, M. J. Frisch, and H. Nakatsuji, J. Phys. Chem. A 109, 3187 (2005).

${ }^{24}$ C. Angeli, M. Pastore, and R. Cimiraglia, Theor. Chem. Acc. 117, 743 (2007).

${ }^{25}$ S. R. Gwaltney and R. J. Bartlett, J. Chem. Phys. 108, 6790 (1998).

${ }^{26}$ In using the term "MBPT" we do not intend the quantum-chemistry approaches that take into account the electronic correlation as a perturbation of Hartree-Fock, but only a subclass of many-body techniques 
developed using a Green's function method in solid state physics, which treat the electron-electron interaction as a perturbation to the homogeneous electron gas.

${ }^{27}$ F. Aryasetiawan and O. Gunnarsson, Rep. Prog. Phys. 61, 237 (1998); W. G. Aulbur, L. W. Jönsson, and J. W. Wilkins, Solid State Phys. 54, 1 (1999) (and references therein).

${ }^{28}$ G. Onida, L. Reining, and A. Rubio, Rev. Mod. Phys. 74, 601 (2002) (and references therein).

${ }^{29}$ Time Dependent Density Functional Theory, Lecture Notes in Physics Vol. 706, edited by M. A. L. Marques, C. A. Ullrich, F. Nogueira, A. Rubio, K. Burke, E. K. U. Gross (Springer, Berlin, Heidelberg, 2006), Chaps. 16-23; Phys. Chem. Chem. Phys. 11, Issue (22) (2009).

${ }^{30}$ J. C. Grossman, M. Rohlfing, L. Mitas, S. G. Louie, and M. L. Cohen, Phys. Rev. Lett. 86, 472 (2001).

${ }^{31}$ M. Rohlfing and S. G. Louie, Phys. Rev. Lett. 80, 3320 (1998).

${ }^{32}$ M. Bruno, M. Palummo, A. Marini, R. Del Sole, and S. Ossicini, Phys. Rev. Lett. 98, 036807 (2007)

${ }^{33}$ P. H. Hahn, W. G. Schmidt, and F. Bechstedt, Phys. Rev. B 72, 245425 (2005).

${ }^{34}$ M. Rohlfing and S. G. Louie, Phys. Rev. Lett. 82, 1959 (1999).

${ }^{35}$ A. Ruini, M. J. Caldas, G. Bussi, and E. Molinari, Phys. Rev. Lett. 88, 206403 (2002).

${ }^{36}$ K. Hummer and C. Ambrosch-Draxl, Phys. Rev. B 71, 081202 (2005).

${ }^{37}$ D. Varsano, A. Marini, and A. Rubio, Phys. Rev. Lett. 101, 133002 (2008).

${ }^{38}$ D. Prezzi, D. Varsano, A. Ruini, A. Marini, and E. Molinari, Phys. Rev. B 77, 041404(R) (2008).

${ }^{39}$ N. Sai, M. L. Tiago, J. R. Chelikowsky, and F. A. Reboredo, Phys. Rev. B 77, 161306 (2008).

${ }^{40}$ A. L. Fetter and J. D. Walecka, Quantum Theory of Many-Particle Systems (Dover, New York, 2003), Chap. 15, p. 565.

${ }^{41}$ M. Grüning, A. Marini, and X. Gonze, "Exciton-Plasmon States in Nanoscale Materials: Breakdown of the Tamm-Dancoff Approximation," Nano Lett. (in press) (e-print arXiv:0909.3389).

${ }^{42}$ Z.-L. Cai, K. Sendt, and J. R. Reimers, J. Chem. Phys. 117, 5543 (2002).

${ }^{43}$ P. Hohenberg and W. Kohn, Phys. Rev. 136, B864 (1964); W. Kohn and L. J. Sham, ibid. 140, A1133 (1965).

${ }^{44}$ J. P. Perdew, K. Burke, and M. Ernzerhof, Phys. Rev. Lett. 77, 3865 (1996).

${ }^{45}$ QUANTUM-ESPRESSO is a community project for high-quality quantumsimulation software, based on density-functional theory, and coordinated by P. Giannozzi. See http://www.quantum-espresso.org and http:// www.pwscf.org.

${ }^{46}$ D. Lamoen and M. Parrinello, Chem. Phys. Lett. 248, 309 (1996).

${ }^{47}$ G. G. Gurzadyan, T.-H. Tran-Thi, and T. Gustavsson, J. Chem. Phys. 108, 385 (1998).
${ }^{48}$ Y.-H. Zhang, W.-J. Ruan, Z.-Y. Li, Y. Wu, and J.-Y. Zheng, Chem. Phys. 315, 201 (2005).

${ }^{49}$ J. P. Perdew and A. Zunger, Phys. Rev. B 23, 5048 (1981).

${ }^{50}$ A. Marini, C. Hogan, M. Grüning, and D. Varsano, Comput. Phys. Commun. 180, 1392 (2009) (the code is freely available from http:// www.yambo-code.org).

${ }^{51}$ S. L. Adler, Phys. Rev. 126, 413 (1962); N. Wiser, ibid. 129, 62 (1963).

${ }^{52}$ C. A. Rozzi, D. Varsano, A. Marini, E. K. U. Gross, and A. Rubio, Phys. Rev. B 73, 205119 (2006).

${ }^{53}$ M. Rohlfing and S. G. Louie, Phys. Rev. B 62, 4927 (2000).

${ }^{54}$ M. Gatti, F. Bruneval, V. Olevano, and L. Reining, Phys. Rev. Lett. 99, 266402 (2007).

${ }^{55}$ S. Galamic-Mulaomerovic and C. H. Patterson, Phys. Rev. B 72, 035127 (2005).

${ }^{56}$ F. Sottile, M. Marsili, V. Olevano, L. Reining, Phys. Rev. B 76, 161103 (2007).

${ }^{57}$ N. P. Wang, M. Rohfing, P. Kruger, and J. Pollman, Phys. Rev. B 71, 045407 (2005).

${ }^{58}$ J. Seda, J. V. Burda, and J. Leszczynski, J. Comput. Chem. 26, 294 (2005).

${ }^{59}$ P. Dupuis, R. Roberge, and C. Sandorfy, Chem. Phys. Lett. 75, 434 (1980).

${ }^{60}$ A. Becke, J. Chem. Phys. 98, 5648 (1993).

${ }^{61}$ A. Ghosh and T. Vangberg, Theor. Chem. Acc. 97, 143 (1997).

${ }^{62}$ N. Dori, M. Menon, L. Kilian, M. Sokolowski, L. Kronik, and E. Umbach, Phys. Rev. B 73, 195208 (2006).

${ }^{63}$ N. Marom, O. Hod, G. E. Scuseria, and L. Kronik, J. Chem. Phys. 128, 164107 (2008).

${ }^{64}$ M. Casida, J. Chem. Phys. 122, 054111 (2005).

${ }^{65}$ M. Gouterman and G. E. Khalil, J. Mol. Spectrosc. 53, 88 (1974).

${ }^{66}$ J. G. Radziszewski, J. Waluk, M. Nepras, and J. Michl, J. Phys. Chem. 95, 1963 (1991).

${ }^{67}$ H. Gratz and A. Penzkofer, Chem. Phys. 254, 363 (2000).

${ }^{68}$ A. Aspuru-Guzik, O. El Akramine, J. C. Grossman, and W. A. Lester, Jr., J. Chem. Phys. 120, 3049 (2004).

${ }^{69}$ Real space-real time calculation, using the code octopus (Ref. 70), within the LDA (GGA gives very similar results, however). BSE and TDDFT spectra were compared using $\sigma(\omega)=\omega / c \operatorname{Im}\left\{\varepsilon^{-1}(\mathbf{q}=0, \omega)\right\}$, where $\sigma$, the photoabsorption cross section, is the quantity calculated by осторus (real-space approach), and $\varepsilon^{-1}(\mathbf{q}=0, \omega)$ is the quantity calculated by YAMBO (plane wave approach). We scaled the BSE spectra such that the $y$ component intensity was equal to that of the TDDFT spectrum.

${ }^{70}$ M. A. L. Marques, A. Castro, G. F. Bertsch, and A. Rubio, Comput. Phys. Commun. 151, 60 (2003).

${ }^{71}$ P. Umari, G. Stenuit, and S. Baroni, Phys. Rev. B 79, 201104 (2009). 